Article

\title{
Hepatobiliary-Related Outcomes in US Adults Exposed to Lead
}

\author{
Emmanuel Obeng-Gyasi ${ }^{1, *}$, Rodrigo X. Armijos ${ }^{2}$, M. Margaret Weigel ${ }^{2}$, Gabriel Filippelli ${ }^{3}$ \\ and M. Aaron Sayegh 4 \\ 1 Department of Built Environment, North Carolina Agricultural and Technical State University; \\ Greensboro, NC 27411, USA \\ 2 Department of Environmental and Occupational Health, Indiana University School of Public Health, \\ Bloomington, IN 47405, USA; rarmijos@indiana.edu (R.X.A.); weigelm@iu.edu (M.M.W.) \\ 3 Department of Earth Sciences, Indiana University Purdue University Indianapolis; \\ Indianapolis, IN 46202, USA; gfilippe@iupui.edu \\ 4 Department of Epidemiology and Biostatistics, Indiana University School of Public Health, \\ Bloomington, IN 47405, USA; msayegh@indiana.edu \\ * Correspondence: eobenggyasi@ncat.edu; Tel.: +1-336-285-3132
}

Received: 28 February 2018; Accepted: 28 March 2018; Published: 31 March 2018 updates

\begin{abstract}
The purpose of this cross-sectional study was to investigate hepatobiliary-related clinical markers in Unites States adults (aged $\geq 20$ ) exposed to lead using the National Health and Nutrition Examination Survey (NHANES) 2007-2008 and 2009-2010 datasets. Clinical markers and occupation were evaluated in 4 quartiles of exposure $-0-2 \mu \mathrm{g} / \mathrm{dL}, 2-5 \mu \mathrm{g} / \mathrm{dL}, 5-10 \mu \mathrm{g} / \mathrm{dL}$, and $10 \mu \mathrm{g} / \mathrm{dL}$ and over-to examine how the markers and various occupations manifested in the quartiles. Linear regression determined associations, and binary logistic regression predicted the likelihood of elevated clinical makers using binary degrees of exposure set at $(2 \mu \mathrm{g} / \mathrm{dL}, 5 \mu \mathrm{g} / \mathrm{dL}$, and $10 \mu \mathrm{g} / \mathrm{dL})$. Clinical makers, and how they manifested between exposed and less-exposed occupations, were explored in addition to how duration of exposure altered these clinical markers. In regression analysis, Gamma-Glutamyl Transferase (GGT), total bilirubin, and Alkaline Phosphatase (ALP) were positively and significantly associated with Blood lead level (BLL). Using binary logistic regression models, at the binary $2 \mu \mathrm{g} / \mathrm{dL}$ level ALP, and GGT were more likely to be elevated in those exposed. At $5 \mu \mathrm{g} / \mathrm{dL}$ level, it was ALP and GGT that were more likely to be elevated in those exposed whereas at $10 \mu \mathrm{g} / \mathrm{dL}$ level, it was GGT that were more likely to be elevated in those exposed. In the occupational analysis, Aspartate Aminotransferase (AST), Alanine Aminotransferase (ALT), GGT, and ALP showed differences between populations in the exposed and less-exposed occupations. Regarding Agriculture, Forestry and Fishing, duration of exposure altered AST, ALP, and total bilirubin significantly $(p<0.05)$ while ALT and GGT were altered moderately significantly $(p<0.10)$. With mining, duration of exposure altered AST and GGT moderately significantly, whereas in construction duration in occupation altered AST, and GGT significantly, and total bilirubin moderately significantly. The study findings are evidence of occupational exposure to lead playing a significant role in initiating and promoting adverse hepatobiliary clinical outcomes in United States adults.
\end{abstract}

Keywords: lead exposure; hepatobiliary outcomes; heavy metals; occupational exposure

\section{Introduction}

Lead exposure among adults mainly occurs in the workplace within lead and zinc ore mining, painting, and battery manufacturing industries [1]. Indeed, occupational exposure to inorganic lead in Western countries occurs in mines and smelters, welding of lead painted metal, battery manufacturing plants, and in the glass manufacturing industry [2]. Exposure to lead can also occur in 
community-based settings as is the case in El Paso, Texas, and Baltimore, Maryland. This is due to the legacy of leaded gasoline and paint [3,4] in addition to industrial sources of exposure [5].

Lead is toxic and causes many adverse clinical outcomes in adults. It has no role in normal biological function in humans but through several mechanisms can induce adverse health outcomes, including adverse hepatobiliary outcomes. Furthermore, because lead is environmentally persistent, populations can remain continuously exposed in areas where it was previously used. Sources and extent of lead exposure have fallen dramatically over the past 30 years because of interventions such as, lead content being reduced in gasoline, lead being limited in household paint, existing sources of lead paint being contained, as well as lead being limited in the food canning process and in industrial emissions and water. Despite that, lead continues to be an issue of public health significance in the United States. It is biologically and environmentally persistent, making it hazardous as it affects almost every organ system within the human body, including the hepatobiliary system [6-9]. Studies on the hepatotoxic effects of lead have demonstrated that exposure to lead alters cholesterol metabolism, xenobiotic metabolism, and plays a role in hepatic hyperplasia [10]. Oxidative stress is a major mechanism for the pathogenesis of lead induced toxicity. Hsu and Leon Guo [11] determined that lead-induced oxidative stress contributes to the pathogenesis of lead poisoning by disrupting the delicate pro-oxidant/antioxidant balance in the cells of mammals. Indeed, lead exposure causes the generation of reactive oxygen species and modification of antioxidant defense systems in occupationally exposed workers and animals and thus negatively impacts the liver. Autopsy studies of lead-exposed patients have shown that a significant proportion of absorbed lead is stored in the liver, giving credence to its disproportionate effects in the hepatobiliary system [12]. Studying more about its effects on liver injury is thus an issue of public health importance as liver diseases affects 3.9 million United States adults and there are 12 deaths per 100,000 people in the United States from liver disease [13].

Liver and biliary system injury can be evaluated by examining clinical markers of liver damage. Liver function test measuring hepatic enzymes such as Alanine Aminotransferase (ALT), Aspartate Aminotransferase (AST), Alkaline Phosphatase Test (ALP), and Gamma-Glutamyl Transferase (GGT), in addition to test measuring Total Bilirubin, help to assess the injury status of the liver and gallbladder, but these clinical markers can also be elevated due to pathology in other organs; thus, an appropriate differential diagnosis needs to be administered [14].

Aminotransferases are used to detect and monitor the progression and resolution of hepatocellular injury [15]. AST catalyzes the conversion of aspartate and alpha-ketoglutarate to oxaloacetate and glutamate [16]. AST is synthesized in the liver but is not specific to the liver: the kidney, heart, brain, and muscles cells also synthesize smaller amounts of AST. ALT catalyzes the transfer of an amino group to alpha-ketoglutarate from alanine in the alanine cycle, producing pyruvate and glutamate in the process [16]. It is synthesized in the liver and is also usually present in low amounts in the blood. With liver injury ALT tends to rise, as it is more specific to the liver than AST. Gamma-glutamyl transferase (GGT) is a sensitive marker of hepatic inflammation. GGT, an enzyme that functions in the gamma-glutamyl cycle, catalyzes the transfer of gamma-glutamyl functional groups from molecules such as glutathione and is found not only in the liver but in other organ tissues, including the kidney and pancreas. Elevated GGT levels in serum may indicate various liver pathologies, including fatty liver disease, liver inflammation or hepatitis [17]. It can also be elevated due to cholestasis. Alkaline phosphatase is found throughout the body, including in the liver, kidney, bone, and digestive system, and is a good marker to test for hepatobiliary damage in addition to bone cell dysregulation. Concentration of ALP is generally increased by cholestasis, injury to intestinal epithelium, or damage to the biliary epithelium [16]. Total Bilirubin consists of unconjugated and conjugated bilirubin. Unconjugated bilirubin is formed when heme is released from hemoglobin and is converted to unconjugated bilirubin, the unconjugated bilirubin is then transported to the liver where within the hepatocytes bilirubin, via a uridine diphosphoglucuronate-glucuronyltransferase (UDP-GT) process, is conjugated with glucuronic acid. This process can be altered by various pathology along 
the pathway. Serum activity of these clinical markers are often a reflection of the physiological state of the liver and their activity in blood often indicates the severity of cellular damage [12,14]. The effects of lead on liver injury was examined in a study that looked at the effects of blood lead on plasma levels of amino acids and serum liver enzymes among the exposed (100 industrial workers) and controls (100-non industrial workers) in which they found liver enzymes to be significantly elevated in industrialized workers as compared to non-industrialized workers [18].

Adult Blood Lead Epidemiology Surveillance (ABLES) operated under the Centers for Disease Control and Prevention (CDC), is a state-based surveillance of adult BLLs in the United States. In 1994, the rate of lead exposure to workers resulting in BLLs $\geq 25 \mu \mathrm{g} / \mathrm{dL}$ was 14 employed adults per 100,000. In 2011, the rate was 6.4 employed adults per 100,000. The Occupational Safety and Health Administration's (OSHA) lead standards require that workers be removed from lead exposure sources when BLLs $\geq 50 \mu \mathrm{g} / \mathrm{dL}$ in the construction industry or $60 \mu \mathrm{g} / \mathrm{dL}$ in general industry, and in the context of previous elevated exposure, workers are allowed to return to work when their BLLs are below $40 \mu \mathrm{g} / \mathrm{dL}$ [1]. It should be noted that the half-life of lead in blood is 35 days and 30 years in bone [19]. Blood lead level (BLL) is a reflection of acute exposure to lead while bone lead is a measure of chronic exposure to lead. Using BLL to measure lead exposure is a validated method in both precision and accuracy and a better method to test lead levels than urine lead measurements, which, because of lead's rapid clearance, makes it less accurate. Our study sought to examine the effects of lead on the hepatobiliary system in the US general adult populations.

\section{Materials and Methods}

\subsection{Hypothesis}

In this study it was hypothesized that exposure to lead adversely affects hepatobiliary functions via adversely affecting liver and biliary enzymes. In that respect this study sought to investigate the effects of lead exposure on the studied participants by analyzing their AST, ALT, ALP, GGT, and total bilirubin clinical markers. The analysis of BLLs and clinical markers within a sample of United States adults determined the extent to which exposure to lead potentially altered the markers in individuals. Lead's impact on occupation was also explored to determine its effects on the clinical makers of interest among those occupationally exposed to lead.

The sociodemographic, behavioral, and anthropometric covariates made it possible to statistically control for factors associated with adverse hepatobiliary outcomes. It also made it possible to make estimations about the contribution of lead to studied participants' hepatobiliary clinical markers.

In all, it was hypothesized that being exposed to lead would be associated with elevated ALT, AST, total bilirubin, ALP, and GGT.

\subsection{Study Design}

\section{Research Design}

Data from NHANES 2007-2010 were used to examine the association between lead and hepatobiliary related markers ALT, AST, ALP, total bilirubin and GGT-in the general United States adult population. The NHANES 2007-2010 survey was conducted by the CDC using a representative sample of the U.S. noninstitutionalized civilian population. Altogether, 12,153 adult subjects $\geq 20$ years were included in this complex multistage, stratified cluster survey in 2007 through 2010, which after factoring in sampling weights represented 217,057,187 people. Of the 12,153 participants, blood lead was measured in 9781 adult subjects representing an estimated 182,052,299 people. For ALT values, 10,992 were measured; this represented 204,454,456 of the population. AST value levels were measured for 10,991, representing 204,424,018 of the population whereas GGT value levels were measured for 10,996 , which represented 204,525,189 of the population. With respect to total bilirubin, the levels measured were for 9397, representing 170,044,349 of the population whereas ALP was measured for 10,996 adults, which represented $204,523,110$ of the population. 


\subsection{Data Collection}

Recruitment

In NHANES, participants are selected using a complex sampling methodology with various clinical makers collected every year from participants who are representative of the non-institutionalized population. For this analysis, 4-year weights for individual probabilities drawn from biomarker data sets were used following the NCHS web tutorial (NCHS 2010a). The sample weights for NHANES 2007-2010 were based on population estimates that incorporated the national census count. NHANES 2007-2010 consisted of a standardized questionnaire administered in the home by a trained interviewer followed by a comprehensive physical examination at a Mobile Examination Center (MEC). The data are freely available from the institution's homepage.

A cross-sectional study, NHANES collects nationally representative data on health outcomes and disease. Methods for demographic, clinical and survey data collection can be found at the NCHS' website $[20,21]$.

\subsection{Quality Control}

\subsubsection{For Demographics}

The computer-assisted personal interview (CAPI) software, that was used to gather the interview data in NHANES 2007-2010, contained data edit and consistency checks which notified the interviewer when the recorded data was erroneous. Information screens provided standardized descriptions of the terminology and concepts that were used in the questionnaires for the interviewer. Data collection was consistently reviewed by NHANES field officers and subsets of participants were re-contacted to ensure accuracy. Finally, several interviews were audio-taped and reviewed by National Center for Health Statistics (NCHS) and contractor staff to ensure quality.

\subsubsection{For Hepatobiliary Markers}

The NHANES 2007-2010 quality assurance/quality control (QA/QC) protocols fulfilled the 1988 Clinical Laboratory Improvement Act. Detailed instructions regarding QA/QC are found in the NHANES Laboratory/Medical Technologists Procedures Manual (LPM). The General Documentation of Laboratory Data file provides detailed QA/QC protocols.

\subsubsection{Biomarkers and Biometric Data in This Study}

The major biomarker of interest in this study is blood lead, which is representative of soft tissue lead and a good measure of body burden and internal dose of lead [22]. BLLs can help one determine the degree of exposure to lead at a snapshot in time. Other biomarkers of interest are AST, ALT, GGT, ALP, and total bilirubin. These are hepatobiliary biomarkers. The hepatobiliary biomarkers have been positively associated with exposure to lead in some studies while others have found no association $[18,23,24]$.

\subsubsection{Instruments and Procedures}

In the study, the biochemistry biomarkers were measured using a Beckman Synchron LX20, Beckman UniCel ${ }^{\circledR}$ DxC800 Synchron (Brea, CA, USA) at Collaborative Laboratory Services and the Roche Modular P chemistry analyzer at the University of Minnesota, MN, USA. Metal assays in whole blood samples were conducted in the NHANES 2007-2010 at the Division of Laboratory Sciences, National Center for Environmental Health (NCEH) of the CDC. Blood lead was determined by inductively coupled plasma mass spectrometry (ICP-MS; CDC method no. ITB0001A). 


\subsection{Data Management}

Data management was done in accordance with the NHANES analytical guidelines relating to its survey design and weighting [25]. The software Stata SE/15.0 (StataCorp, College Station, TX, USA) was used for data management.

\subsection{Analytical and Statistical Approaches}

This study analyzed results from adults aged 20 and older. In portions of the study, analysis was performed on those experiencing various degrees of exposure represented by BLLs in four quartiles; $0-2 \mu \mathrm{g} / \mathrm{dL}, 2-5 \mu \mathrm{g} / \mathrm{dL}, 5-10 \mu \mathrm{g} / \mathrm{dL}, 10+\mu \mathrm{g} / \mathrm{dL}$ presented in this study as quartile 1 , quartile 2 , quartile 3 , and quartile 4 respectively, which represent thresholds typically and historically used in the literature to represent elevated exposure. Association between lead and hepatobiliary outcomes were explored using linear regression. Since the variables of interest were not normally distributed, natural $\log$ transformation was used for dependent and independent variables in regression analysis.

Both continuous and categorical data were analyzed. For linear regression, all independent variables were examined as continuous variables. The covariates of interest (gender, BMI, ethnicity, and age), and consumption of alcohol (those who had taken at least 12 alcoholic drinks in the past year) and smoking habits, were adjusted for to determine leads impact on the clinical markers of interest.

In the binary logistic regression models, the dependent variable was categorical for lead exposure at the 2,5, and $10 \mu \mathrm{g} / \mathrm{dL}$ levels. Statistical analyses were performed using Stata SE/15.0 (StataCorp, College Station, TX, USA) as the software allowed for adjustment for clusters and strata of the complex sample in addition to incorporating the sample weight in order to generate estimates for the total noninstitutionalized civilian population of the United States.

In addition, occupational analysis was performed examining the mean difference between the clinical markers of occupationally exposed workers as compared to occupationally less exposed workers. Finally, the mean levels of the clinical markers of interest were explored in durations of $0-5$ years, $5-10$ years, and over 10 years to see how the markers manifested over various time periods.

A $p$-value of $<0.05$ was considered significant while a value of $<0.10$ was considered moderately significant. Excel 2016 was used to generate charts/figures.

\section{Results}

\subsection{Sociodemographic, Anthropometric and Clinical Variables/Data}

The objective of this section is a presentation of the results on lead exposure with regards to the participant sociodemographic characteristics, and those of their clinical and anthropometric information. The results of the sociodemographic information will be presented first. In presenting the results of participants' sociodemographic information; gender and ethnicity, as well as their occupation are presented.

\subsubsection{Gender}

The results (in percentages) of the different gender categories and quartiles of exposure in the study data are shown in Table 1 below with the percent standard error (SE\%), a measure of the precision of the mean, shown in brackets. As can be seen, males represent a larger percent of those in the highest exposure groups while females represent a larger percent of the lowest exposure group. 
Table 1. Gender and quartiles of exposure.

\begin{tabular}{ccc}
\hline Lead Exposure & Male\% (SE\%) & Female\% (SE\%) \\
\hline $\mathrm{N}$ & 5858 & 6295 \\
Quartile 1 $(0-2 \mu \mathrm{g} / \mathrm{dL})$ & $67.8 \%(1.2)$ & $82.5 \%(0.9)^{* * * *}$ \\
Quartile 2 $(2-5 \mu \mathrm{g} / \mathrm{dL})$ & $27.8 \%(1.0)^{* *}$ & $16.6 \%(0.8)$ \\
Quartile 3 $(5-10 \mu \mathrm{g} / \mathrm{dL})$ & $3.7 \%(0.3)^{* * *}$ & $0.9 \%(0.1)$ \\
Quartile 4 $(10+\mu \mathrm{g} / \mathrm{dL})$ & $0.7 \%(0.2)^{*}$ & $0.1 \%(0.0)$ \\
Total & $100 \%$ & $100 \%$
\end{tabular}

${ }^{*} p<0.05$ Male making up a significantly larger proportion than Female in this exposure group; ${ }^{* * *} p<0.05$ Male making up a significantly larger proportion than Female in this exposure group; ${ }^{* *} p<0.05$ Male making up a significantly larger proportion than Female in this exposure group; ${ }^{* * * *} p<0.05$ Female making up a significantly larger proportion than Male in this exposure group.

\subsubsection{Ethnicity}

The ethnic groups identified in the data were Mexican American, Non-Hispanic White, Non-Hispanic Black, Other Hispanic, and Other Race (including multi-racial). Percentages representing the various quartiles of exposure are shown in Table 2 below. As can be seen, among Mexican-Americans, a larger percentage are in the highest exposure group (quartile 4) as compared to other ethnic groups. While in the second largest exposure group, Blacks and Mexican-Americans make up a larger percentage as compared to other ethnic groups.

Table 2. Ethnicity and quartiles of exposure.

\begin{tabular}{|c|c|c|c|c|c|}
\hline Lead Exposure & $\begin{array}{c}\text { Mexican } \\
\text { American\% (SE\%) }\end{array}$ & $\begin{array}{l}\text { Other Hispanic\% } \\
\text { (SE\%) }\end{array}$ & $\begin{array}{l}\text { Non-Hispanic } \\
\text { White\% (SE\%) }\end{array}$ & $\begin{array}{l}\text { Non-Hispanic } \\
\text { Black\% (SE\%) }\end{array}$ & $\begin{array}{l}\text { Other Race-Including } \\
\text { Multi-Racial\% (SE\%) }\end{array}$ \\
\hline$N$ & 1032 & 600 & 8342 & 1377 & 802 \\
\hline \multicolumn{6}{|l|}{$0-2 \mu \mathrm{g} / \mathrm{dL}$} \\
\hline Quartile 2 & $23.2 \%(1.4)^{++}$ & $17.3 \%(2.4)$ & $21.1 \%(1.1)$ & $24.4 \%(1.2)^{+}$ & $29.7 \%(2.9) * * * *$ \\
\hline \multicolumn{6}{|l|}{$2-5 \mu \mathrm{g} / \mathrm{dL}$} \\
\hline Quartile 4 & $1.0 \%(0.3) *, \#$ & $0.4 \%(0.3)$ & $0.2 \%(0.1)$ & $0.3 \%(0.1)$ & $0.8 \%(0.5)$ \\
\hline \multicolumn{6}{|l|}{$10+\mu \mathrm{g} / \mathrm{dL}$} \\
\hline Total & $100 \%$ & $100 \%$ & $100 \%$ & $100 \%$ & $100 \%$ \\
\hline
\end{tabular}

${ }^{+} p<0.05$ significant difference between Non-Hispanic Black and Other Hispanic and non-Hispanic White; ${ }^{++} p<0.05$ significant difference between Mexican-American and Other Hispanic; ${ }^{+++} p<0.05$ significant difference between Other Hispanic and Mexican-American, non-Hispanic Black and Other Race-including multi-racial; * $p<0.05$ significant difference between Mexican American and Non-Hispanic white; ${ }^{* *} p<0.05$ significant difference between Mexican American and other Hispanic and non-Hispanic white; ${ }^{* * *} p<0.05$ significant difference between non-Hispanic Black and Other Hispanic, non-Hispanic White; ${ }^{* * * *} p<0.05$ significant difference between other raceincluding multiracial and Mexican American, Other Hispanic, and non-Hispanic white; ${ }^{* * * *} p<0.05$ significant difference between non-Hispanic White and Mexican-American, Non-Hispanic Black and Other Race-Including Multiracial; ${ }^{\#} p<0.10$ moderately significant difference between Mexican American and Non-Hispanic Black.

\subsubsection{Occupation}

Longevity in Jobs (Occupation One Had Spent the Most Time in)

The longevity of employment in a job was also taken into consideration to see how it manifested across different quartiles of exposure. Table 3 summarizes the results. As can be seen Agriculture, Forestry, and Fishing, Mining, and Construction make up a large percentage of the highest exposure groups. 
Table 3. Longest held occupation and quartiles of exposure.

\begin{tabular}{|c|c|c|c|c|}
\hline Occupation & Quartile 1 & Quartile 2 & Quartile 3 & Quartile 4 \\
\hline Accommodation, Food Services & $80.8 \%(2.7)$ & $17.7 \%(2.7)$ & $1.4 \%(0.5)$ & $0.2 \%(0.2)$ \\
\hline Arts, Entertainment, Recreation & $82.4 \%(4.0)^{* * *}$ & $16.8 \%(3.9)$ & $0.8 \%(0.8)$ & $-\mathrm{a}$ \\
\hline Construction & $58.2 \%(3.0)$ & $35.4 \%(2.7) * *$ & $5.1 \%(1.1)^{* * * *}$ & $1.3 \%(0.5) *$ \\
\hline Education Services & $69.1 \%(3.2)$ & $29.6 \%(3.1)$ & $1.4 \%(0.5)$ & $-\mathrm{a}$ \\
\hline Health Care, Social Assistance & $78.1 \%(2.4)$ & $20.0 \%(2.5)$ & $1.8 \%(0.6)$ & $0.01 \%(0.0)$ \\
\hline Information & $71.7 \%(4.3)$ & $27.6 \%(4.2)$ & $0.2 \%(0.2)$ & $0.5 \%(0.5)$ \\
\hline Management/Waste Services & $77.7 \%(4.3)$ & $21.1 \%(4.3)$ & $1.2 \%(0.9)$ & $-a$ \\
\hline Manufacturing: Durable Goods & $66.3 \%(2.3)$ & $28.6 \%(2.1)$ & $4.4 \%(0.7)$ & $0.8 \%(0.5) *$ \\
\hline Manufacturing: Non-Durable Goods & $68.6 \%(2.6)$ & $28.4 \%(2.4)$ & $2.8 \%(0.8)$ & $0.3 \%(0.2)$ \\
\hline Mining & $53.7 \%(9.9)$ & $38.7 \%(7.3) * *$ & $7.6 \%(2.9)^{* * * *}$ & $-a$ \\
\hline Public Administration & $68.8 \%(3.2)$ & $29.4 \%(3.1)$ & $1.0 \%(0.6)$ & $0.8 \%(0.8)$ \\
\hline Real Estate, Rental, Leasing & $83.0 \%(5.4)^{* * *}$ & $13.8 \%(4.8)$ & $2.7 \%(1.8)$ & $0.4 \%(0.4)$ \\
\hline Retail Trade & $79.1 \%(2.5)$ & $18.3 \%(2.2)$ & $2.0 \%(0.7)$ & $0.5 \%(0.5)$ \\
\hline Transportation, Warehousing & $62.2 \%(4.5)$ & $34.4 \%(4.3)$ & $3.5 \%(1.1)$ & \\
\hline Utilities & $51.6 \%(8.4)$ & $43.1 \%(8.2)^{* *}$ & $5.4 \%(4.5)^{* * * *}$ & $-a$ \\
\hline Wholesale Trade & $75.0 \%(4.0)$ & $18.8 \%(3.5)$ & $6.1 \%(2.2)$ & $-a$ \\
\hline
\end{tabular}

-a, no data; ${ }^{*} p<0.05$ for Construction and Manufacturing Durable Goods representing a significantly larger proportion of exposure category as compared to many other industries in the category such as Finance, Insurance, and Health Care, Social Assistance; ${ }^{* *} p<0.05$ of Agriculture, Forestry, Fishing, Mining, Utilities and Construction representing a significantly larger proportion of exposure category as compared to many other industries in exposure category such as Professional, Technical Services and Arts, Entertainment, Recreation; *** $p<0.05$ Real Estate, Rental, Leasing; Professional, Technical Services; Arts, Entertainment, Recreation, Private Households representing a significantly larger proportion of exposure category as compared to many other industries in this category, such as agriculture and construction; ${ }^{* * *} p<0.05$ for Agriculture, Forestry, Fishing, Mining, Utilities, and Construction representing a significantly larger proportion of exposure category as compared to many other industries in the exposure category, such as Information and Professional, Technical Services.

\subsection{Age, BMI, and Clinical Markers}

The objective of this section is a presentation of the results of the clinical and anthropometric markers. Specifically, this study section will explore lead's relationship with, age, BMI, AST, ALT, ALP, GGT, and Total Bilirubin. Information by degree of exposure is presented in Table 4. As can be seen, higher exposure resulted in elevated hepatobiliary clinical markers.

Table 4. Age and BMI and quartiles of exposure.

\begin{tabular}{ccccc}
\hline \multirow{2}{*}{ Variables } & Quartile 1 & Quartile 2 & Quartile 3 & Quartile 4 \\
\cline { 2 - 5 } & $\mathbf{0 - 2} \boldsymbol{\mu g} / \mathbf{d L}$ & $\mathbf{2 - 5} \boldsymbol{\mu g} / \mathbf{d L}$ & $\mathbf{5 - 1 0 ~} \mathbf{g g} / \mathbf{d L}$ & $\mathbf{1 0 +} \boldsymbol{\mu g} / \mathbf{d L}$ \\
\hline BLL & $1.09(0.01)$ & $2.78(0.02)$ & $6.40(0.10)$ & $16.11(1.40)$ \\
Age $^{*}$ & $44.25(0.32)$ & $56.05(0.54)$ & $54.77(1.13)$ & $47.56(2.56)$ \\
BMI & $25.79(0.17)$ & $25.91(0.18)$ & $26.06(0.49)$ & $26.40(0.92)$ \\
AST **** & $25.67(0.20)$ & $27.17(0.38)$ & $27.34(1.04)$ & $31.50(5.30)$ \\
ALT & $25.87(0.24)$ & $26.21(0.46)$ & $26.23(1.27)$ & $28.84(2.09)$ \\
ALP ** & $65.68(0.39)$ & $71.72(0.56)$ & $77.42(1.95)$ & $80.60(4.07)$ \\
GGT *** & $26.54(0.40)$ & $33.10(0.92)$ & $40.70(3.77)$ & $41.49(4.99)$ \\
Total Bilirubin & $0.77(0.01)$ & $0.78(0.01)$ & $0.81(0.03)$ & $0.84(0.07)$ \\
\hline
\end{tabular}

* Significant difference between quartile 1 and 2, 3; ** $p<0.05$ significant difference between quartile 1 and 2, 3; ${ }^{* * *} p<0.05$ significant difference between quartile 1 and 2,$3 ;{ }^{* * * *} p<0.05$ significant difference between quartile 1 and 2. 


\subsection{Association of BLL with Clinical Markers of Interest of All Adults}

All variables were adjusted for age, gender, race/ethnicity, BMI, alcohol consumption, and smoking. The associations of BLL, presented as the natural log of BLL $(\operatorname{lnBPb})$, and the hepatobiliary clinical variables are presented in Table 5 below.

Table 5. Associations of BLL hepatobiliary-related markers of interest.

\begin{tabular}{ccc}
\hline Variables & $\operatorname{lnBPb}$ Adjusted $(\mathbf{9 5} \% \mathbf{C I})^{+}$ & $p$ Value \\
\hline AST & $0.023(-0.057,0.103)$ & 0.568 \\
ALT & $-0.035(-0.083,0.013)$ & 0.147 \\
ALP & $0.264(0.206,0.321)$ & 0.0001 \\
GGT & $0.073(0.046,0.099)$ & 0.0001 \\
Total Bilirubin & $0.079(0.025,0.133)$ & 0.004 \\
\hline${ }^{+}$Adjusted for age, gender, race/ethnicity, BMI, alcohol consumption and smoking.
\end{tabular}

\subsection{Binary Logistic Regression Analysis of Clinical Makers}

Binary logistic regression analysis was performed to examine the factors associated with BLLs at different levels of exposure. Detailed results of hepatobiliary related factors are shown in Table 6.

Table 6. Logistic regression analysis BLL binary at $2 \mu \mathrm{g} / \mathrm{dL}, 5 \mu \mathrm{g} / \mathrm{dL}$, and $10 \mu \mathrm{g} / \mathrm{dL}$ level.

\begin{tabular}{|c|c|c|c|}
\hline \multirow{2}{*}{ Variable in Model } & Binary at $2 \mu \mathrm{g} / \mathrm{dL}$ & Binary at $5 \mu \mathrm{g} / \mathrm{dL}$ & Binary at $10 \mu \mathrm{g} / \mathrm{dL}$ \\
\hline & Adj. Odds Ratio $(95 \% \mathrm{CI})^{+}$ & Adj. Odds Ratio $(95 \% \mathrm{CI})^{+}$ & Adj. Odds Ratio $(95 \% \text { CI })^{+}$ \\
\hline ALT & $0.858(0.707,1.04)$ & $0.849(0.529,1.36)$ & $1.36(0.677,2.74)$ \\
\hline AST & $0.982(0.736,1.31)$ & $1.12(0.568,2.21)$ & $2.10(0.520,8.48)$ \\
\hline ALP & $1.93(1.42,2.612) *$ & $2.90(1.18,7.08)$ * & $4.23(0.576,31.1)$ \\
\hline GGT & $1.24(1.08,1.42) *$ & $1.48(1.12,1.95)$ * & $2.19(1.34,3.56)$ * \\
\hline Total Bilirubin & $1.05(0.770,1.42)$ & $1.36(0.644,2.85)$ & $2.43(0.543,10.9)$ \\
\hline
\end{tabular}

${ }^{+}$Adjusted for age, gender race/ethnicity and BMI and alcohol consumption and smoking. ${ }^{*} p<0.05$.

\subsection{Occupational Exposure to Lead}

Occupational exposure to lead was explored since most adults are exposed to lead at the workplace. Firstly, it was determined which three occupations had the highest and lowest BLLs at the occupation of longest duration, as BLL is a marker of the exposure level. This was done to examine how the clinical makers of interest manifested in those in high exposure occupations when compared to those in low exposure occupations. Finally, the duration of work was examined in time intervals of $0-5$ years, 5-10 years, and over 10 years to see how length of time at a lead exposed job may alter clinical outcomes of interest.

\section{Occupations Providing Highest Exposure}

The occupations providing a high level of exposure as measured by mean BLLs were: (a) Agriculture, Forestry \& Fishing $2.19 \mu \mathrm{g} / \mathrm{dL}(\mathrm{SE}=0.118$ ); (b) Mining $2.33 \mu \mathrm{g} / \mathrm{dL}(\mathrm{SE}=0.34$ ); and (c) Construction $2.39 \mu \mathrm{g} / \mathrm{dL}$ (SE = 0.12). The occupation providing the lowest levels of exposure as measured by mean BLLs were: (a) Professional, Scientific, Technical Services $1.35 \mu \mathrm{g} / \mathrm{dL}$ (SE = 0.05); (b) Private Household $1.35 \mu \mathrm{g} / \mathrm{dL}$ (SE = 0.11); and (c) Arts, Entertainment, Recreation $1.33 \mu \mathrm{g} / \mathrm{dL}$ $(\mathrm{SE}=0.11)$. These occupations were examined to see the effects of long term exposure on makers of interest via looking at those who had these jobs as their job of longest duration. Figure 1 below illustrates the findings. 


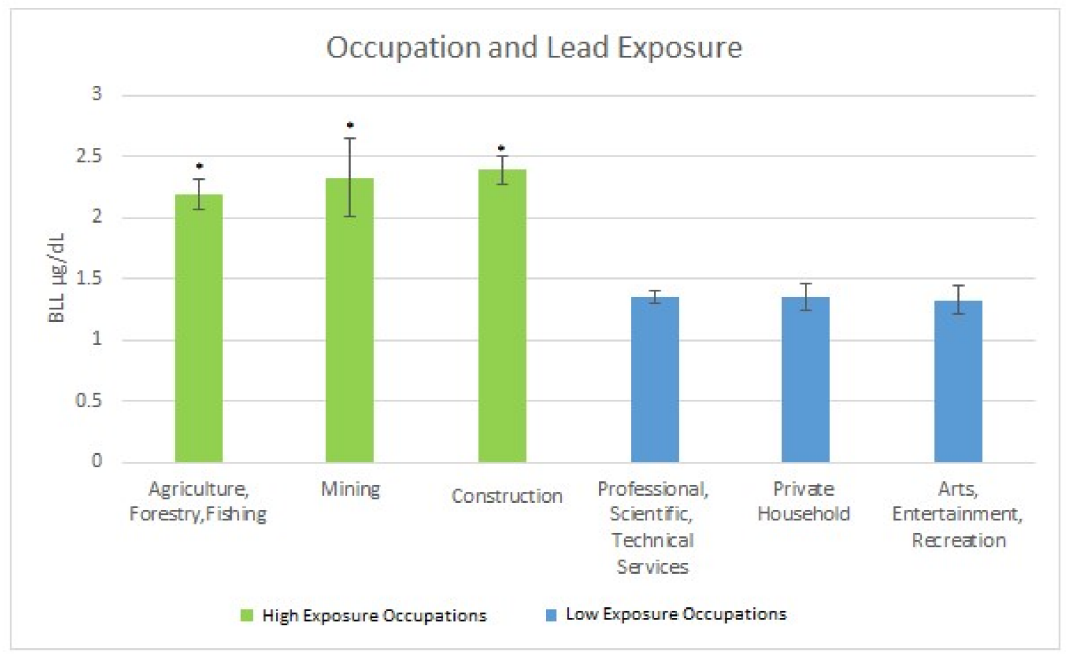

Figure 1. Occupation and Lead Exposure. * Significant difference between high exposure occupations as compared to low exposure occupations.

It is observed that for the high exposure occupations, the mean AST levels were as follows: for Agriculture, Forestry \& Fishing, it was 28.96 U/L (SE = 2.44); for Mining, the level was $28.02 \mathrm{U} / \mathrm{L}$ ( $\mathrm{SE}=1.29$ ); and for Construction, it was $27.86 \mathrm{U} / \mathrm{L}(\mathrm{SE}=0.65)$. For the low exposure occupations, the mean AST levels were: Professional Scientific, Technical Services, $25.81 \mathrm{U} / \mathrm{L}$ (SE = 1.11); Private Household, 25.46 U/L ( $\mathrm{SE}=1.30$ ); and Arts Entertainment, Recreation, 29.40 U/L (SE = 2.16). The mean AST levels are shown in Figure 2 below.

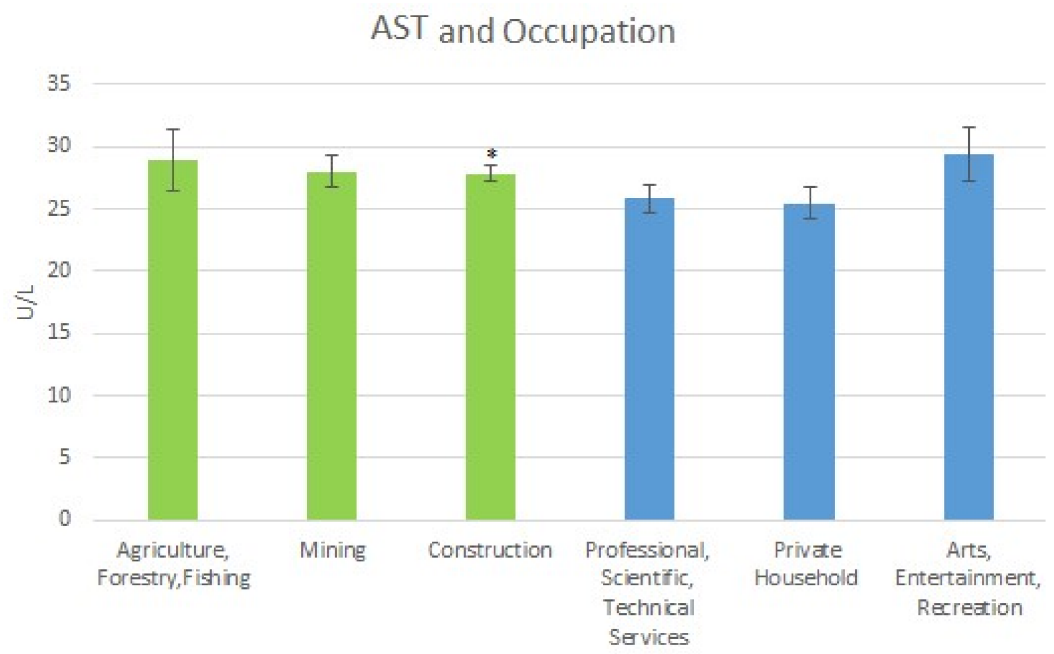

Figure 2. Occupation and Mean AST Levels. * Moderately Significant difference between Construction and Private Households.

For the high exposure occupations, the following mean ALT level were obtained. For Agriculture, Forestry \& Fishing the level was 2.67 U/L ( $\mathrm{SE}=2.54$ ); for Mining it was 28.75 U/L (SE = 1.66); and for Construction, the level was $28.63 \mathrm{U} / \mathrm{L}(\mathrm{SE}=0.85)$. For the low exposure occupations, the mean ALT levels were: Professional Scientific, Technical Services 23.58 U/L (SE = 0.87); Private Household 24.19 U/L (SE = 1.87); and Arts Entertainment, Recreation 27.21 U/L (SE = 2.41). The mean ALT levels are shown in Figure 3 below. 


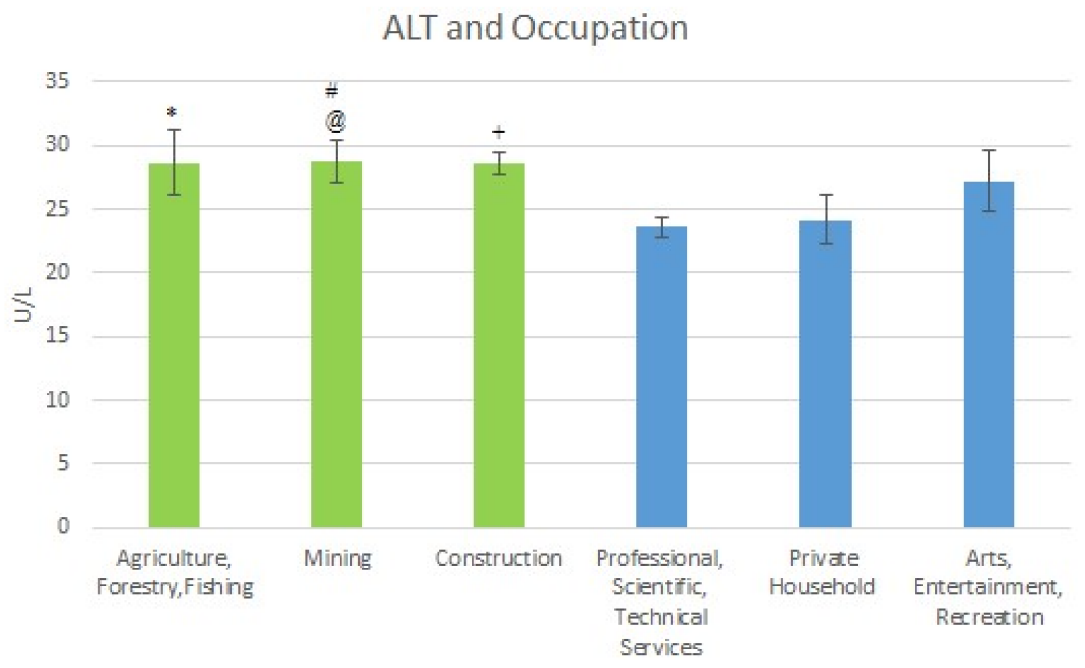

Figure 3. Occupation and Mean ALT Levels. ${ }^{*}$ Moderately significant difference between Agriculture and Professional Scientific, Technical Services. \# Significant difference between Mining and Professional Scientific, Technical Services. @ Moderately significant difference between Mining and Private Households. + Significant difference between Construction and Professional Scientific, Technical Services, and Private Households.

With respect to the high exposure occupations, the following results were obtained for mean GGT levels. For Agriculture, Forestry \& Fishing the GGT level was 30.36 U/L (SE = 1.82); it was $37.41 \mathrm{U} / \mathrm{L}$ $(\mathrm{SE}=2.25)$ for Mining; and 34.75 U/L (SE = 1.97) for Construction. For the low exposure occupations, the mean GGT levels were: Professional Scientific, Technical Services 24.65 U/L (SE = 2.10); Private Household 30.30 U/L (SE = 3.95); and Arts Entertainment, Recreation 27.44 U/L (SE = 3.54). The mean GGT levels are shown in Figure 4 below.

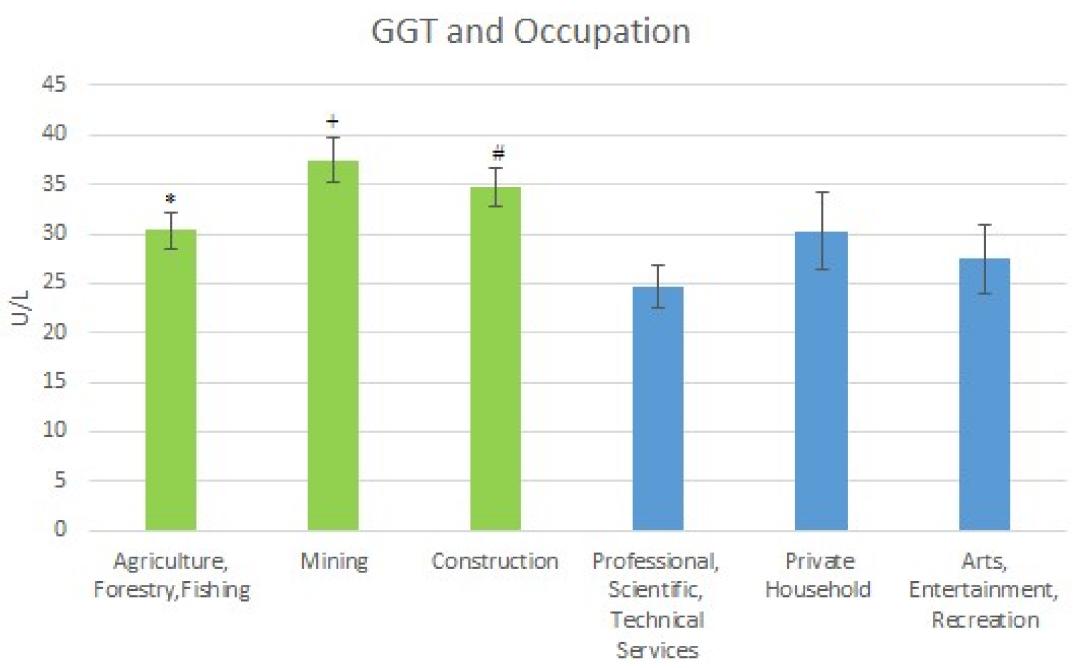

Figure 4. Occupation and Mean GGT Levels. * Significant difference between Agriculture Forestry, Fishing and Professional Scientific, Technical Services. ${ }^{+}$Significant difference between Mining and Professional Scientific, Technical Services and Arts, Entertainment Recreation. \# Significant difference between construction and professional.

Results of the mean total bilirubin levels for the high exposure and low exposure occupations were also obtained. For the high exposure occupations, the results were as follows: for Agriculture, Forestry \& Fishing, it was $0.79 \mathrm{mg} / \mathrm{dL}$ (SE = 0.02); for Mining, the mean total bilirubin level was $0.74 \mathrm{mg} / \mathrm{dL}$ $(\mathrm{SE}=0.03)$; and for Construction it was $0.79 \mathrm{mg} / \mathrm{dL}(\mathrm{SE}=0.03)$. For the low exposure occupations, 
the mean total bilirubin levels were: Professional Scientific, Technical Services, $0.80 \mathrm{mg} / \mathrm{dL}(\mathrm{SE}=0.04)$; Private Household, $0.73 \mathrm{mg} / \mathrm{dL}(\mathrm{SE}=0.04)$; and Arts Entertainment, Recreation, $0.80 \mathrm{mg} / \mathrm{dL}(\mathrm{SE}=0.04)$. The mean total bilirubin levels are shown in Figure 5 below.

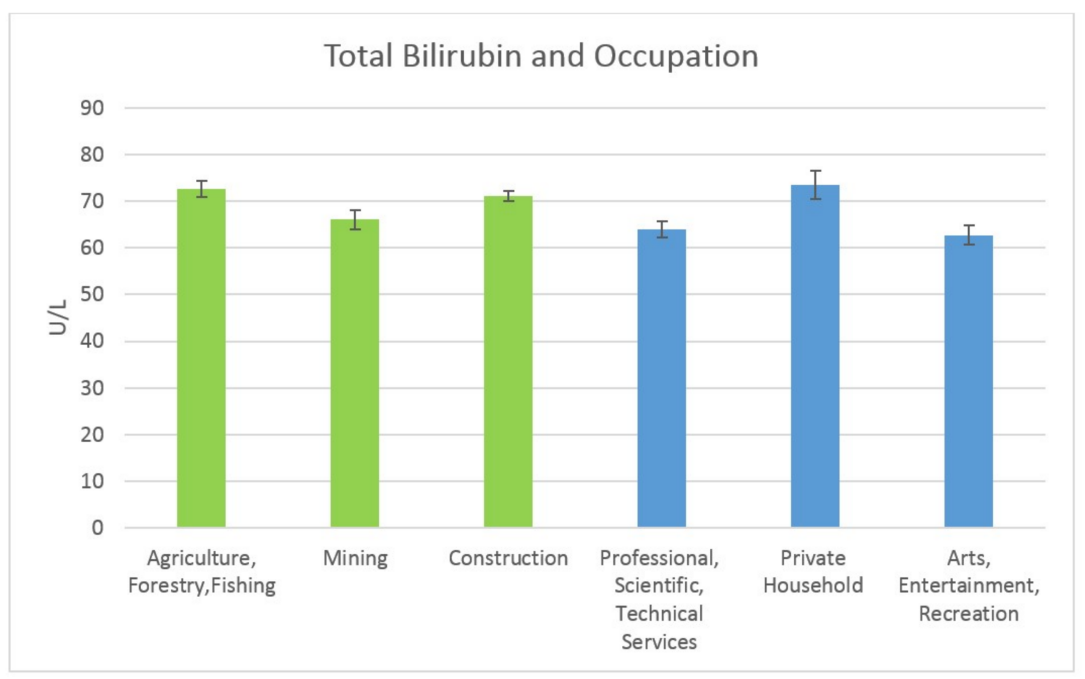

Figure 5. Occupation and Total Bilirubin Levels.

Results of the mean Alkaline Phosphatase (ALP) levels for the high exposure and low exposure occupations were also obtained. For the high exposure occupations, the results were as follows: for Agriculture, Forestry \& Fishing the ALP level was 72.66 U/L (SE = 1.67); for Mining, it was 66.02 U/L $(\mathrm{SE}=2.00)$; and for Construction, the level was 71.13 U/L ( $\mathrm{SE}=1.06)$. For the low exposure occupations, the mean Alkaline Phosphatase levels were: Professional Scientific \& Technical Services, 64.01 U/L (SE = 1.73); Private Household, 73.47 U/L (SE = 3.12); and Arts Entertainment \& Recreation, 62.68 U/L $(\mathrm{SE}=2.05)$. The mean Alkaline Phosphatase levels are shown in Figure 6 below.

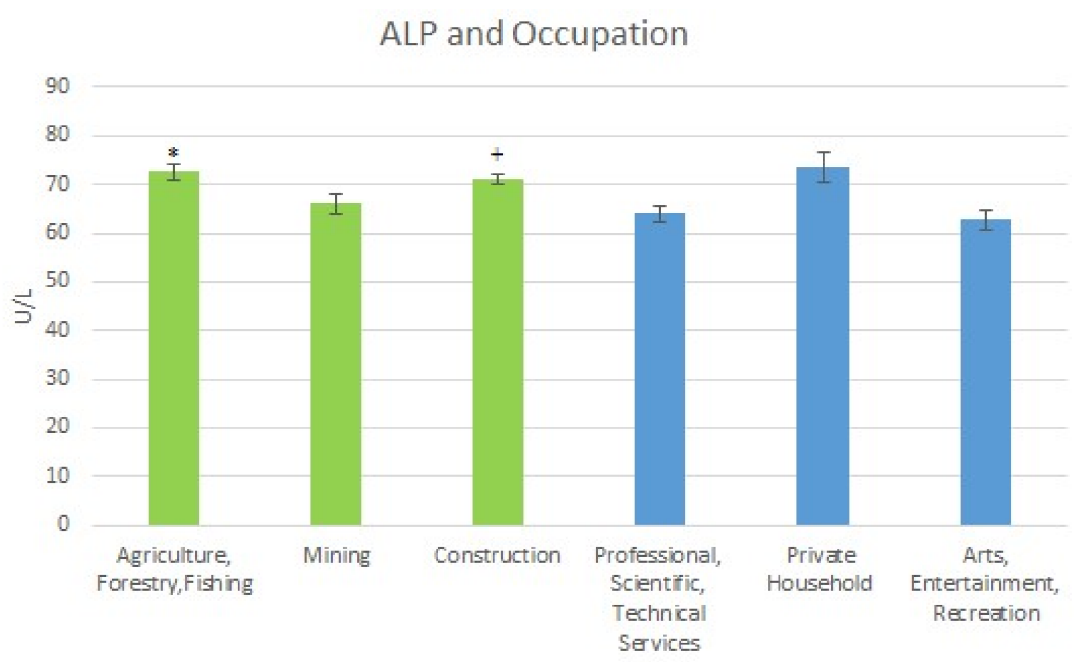

Figure 6. Occupation and Mean ALP Levels. * Significant difference between agriculture and professional and arts. ${ }^{+}$Significant difference between construction and professional and arts.

Finally, the duration of exposure was explored. Length of exposure was examined in three intervals, $0-5$ years of work, 5-10 years of work, and over 10 years of work in the three high exposure occupations of interest in order to better understand the impact of longevity of exposure on the markers of interest. Table 7 reports the mean clinical markers of interest and corresponding standard error (SE). 
Table 7. Longevity in occupation and clinical markers.

\begin{tabular}{|c|c|c|c|c|c|c|c|}
\hline $\begin{array}{c}\text { Time } \\
\text { Interval }\end{array}$ & Occupation & BLL (SE) & AST (SE) & ALT (SE) & ALP (SE) & GGT (SE) & $\begin{array}{l}\text { Total } \\
\text { Bilirubin } \\
\quad \text { (SE) }\end{array}$ \\
\hline $0-5$ years & Construction & $1.58(0.76)$ & $25.54(0.81)$ & $26.97(1.61)$ & $73.62(2.56)$ & $28.40(2.79)$ & $0.86(0.07)$ \\
\hline 5-10 years & Construction & $2.71(0.69)$ & $27.72(1.38)$ & $30.72(2.70)$ & 71.10 (1.82) & 35.78 (5.27) & $0.71(0.04)$ \\
\hline $10+$ years & Construction & $2.53(0.27){ }^{@,} \mathrm{M} 1$ & $28.49(0.78)^{@}$ & $28.60(0.90)$ & 70.49 (1.29) & $36.17(2.30)^{@}$ & $0.79(0.03) \mathrm{M} 2$ \\
\hline $0-5$ years & Mining & $1.05(0.43)$ & $23.03(2.19)$ & $22.61(2.60)$ & $66.81(3.66)$ & $26.47(1.42)$ & $0.81(0.16)$ \\
\hline $5-10$ years & Mining & $1.61(0.28)$ & $33.27(4.93)^{\mathrm{M}}$ & $35.62(6.34)$ & $66.92(2.74)$ & 50.77 (11.1) ${ }^{\mathrm{M} 1}$ & $0.71(0.08)$ \\
\hline $10+$ years & Mining & $2.57(0.38)^{+, @ ~}$ & $27.04(1.57)$ & $27.43(2.38)$ & $65.72(1.98)$ & $34.75(3.33)$ & $0.74(0.03)$ \\
\hline $0-5$ years & Agriculture & $1.72(0.38)$ & $21.65(0.51)$ & $23.89(2.40)$ & $61.96(4.07)$ & $24.39(3.52)$ & $0.64(0.05)$ \\
\hline $5-10$ years & Agriculture & $2.18(0.48)$ & $22.21(0.90)$ & $21.86(2.52)$ & $69.82(6.15)$ & $24.55(3.56)$ & $0.62(0.06)$ \\
\hline $10+$ years & Agriculture & $2.25(0.12)$ & $\begin{array}{c}30.89(3.20){ }^{@}, \\
\end{array}$ & $\begin{array}{c}30.27(3.25) \\
\text { M2 }\end{array}$ & 74.52 (1.92) & 31.89 (M1,2) & $\begin{array}{c}0.83(0.03) \\
@,+\end{array}$ \\
\hline
\end{tabular}

${ }^{\circledR}$ Significantly different from $0-5$ years to $10+; *$ Significantly different $5-10$ years to $10+;{ }^{+}$Significantly difference $0-5$ to $5-10 ;{ }^{M}$ moderately significant difference between $0-5$ years and $5-10$ years; ${ }^{M} 1$ moderately significant difference between $0-5$ years and $10+$ years; $^{\mathrm{M} 2}$ moderately significant difference between $5-10$ years to $10+$ years.

\section{Discussion}

\subsection{On Lead and Occupation}

Cognizant of the fact that lead exposure in adults most commonly occurs in the workplace and industries such as the construction industry, which has historically been a source of lead exposure among adults [26], this study sough to explore occupation and its connection with lead exposure by examining participant hepatobiliary markers in occupations. The results indicated that the quartile of exposure was related to occupation, with occupations such as mining and construction making up a significantly larger proportion of the highest exposure quartiles $(10+\mu \mathrm{g} / \mathrm{dL})$ while occupations such as education made up a significantly larger proportion of the lowest quartiles of exposure $(0-2 \mu \mathrm{g} / \mathrm{dL})$. In subsequent analysis it was determined that the occupations providing the most exposure, as measured by BLLs in adults, were the Construction Industry; Agriculture Forestry \& Fishing; and Mining. The occupations with the least exposed population were: Professional, Scientific and Technical Services; Private Household; and Arts, Entertainment and Recreation. These industries were examined to see how the clinical markers of interest varied between the high exposure occupations and the lower exposure occupation.

In comparing high exposure occupations to low exposure occupations, the mean BLL's were significantly higher when comparing the three high exposure occupations to the three low exposure occupations, affirming that some occupations predispose workers to lead exposure. For example, those in Construction had significantly elevated blood pressure in the highest quartile of exposure as compared to those in the less-exposed occupations such as Education. This is significant as it offers a means to perform targeted intervention toward higher exposure occupations. This exposure and how it manifests cannot only be tied to occupation and may also include behavioral patterns associated with occupations which may accelerate the negative impact of lead on the hepatic and biliary system. Some of these behaviors, including smoking and alcohol use, were adjusted for in this study in order to offer more insight into the extent to which lead may be inducing liver damage. In addition gender and age are also potential factors altering the manifestation of these enzymes [27], hence the need to adjust for them.

For AST, a clinical marker of liver injury, there was a significant difference in the mean levels between those in Construction when compared with those in Private Households, potentially indicating that those in a lead exposed industry experience worse outcomes than those in a less exposed industry. For ALT, there was a significant difference in the mean levels between those in the Mining occupation and those in the Professional, Scientific \& Technical Services, also supporting this conclusion. There was also a moderately significant difference in the mean levels between those in Mining and those in Private Households. Also, a moderately significant difference between the populations in Agriculture Forestry \& Fishing compared with those in Professional Scientific and Technical services was discovered. 
Thus, those lead exposed industries seem to experience elevated liver injury as compared to those in less lead exposed industries.

With respect to GGT, there was a significant difference between Agriculture, Forestry \& Fishing when compared to Professional Scientific, Technical Services. In addition, there was a significant elevation in the mean level of those in Mining when compared to those in Professional Scientific \& Technical Services, and Arts, Entertainment \& Recreation. Finally, there was a significant elevation in construction when compared to Professional, Scientific, and Technical Services, which confirmed the pattern. For Alkaline Phosphatase, there was a significant elevation between Agriculture, Forestry \& Fishing, and Construction when compared to Professional Scientific, Technical Services, and Arts, Entertainment, and Recreation, also confirming lead's potential impact on the hepatic and biliary system.

Finally, in the longest held occupations, a marker of long term exposure to lead due to occupation, results for Construction demonstrated that mean BLLs were significantly elevated from the 0-5-year working period when compared to the 10 plus year working period, while it was moderately elevated from the $0-5$ to 5-10-year period. In Mining, BLLs were significantly elevated from the $0-5$-year to the $10+$ year period and from the $0-5$-year period to the 5-10-year period. Hinting at the potential adverse outcomes over time with hepatobiliary injury becoming more severe over time.

Regarding duration of exposure, AST was moderately elevated from the $0-5$ to the $5-10$-year period for Mining. For Construction, AST was significantly elevated from the 5-10-year to the 10+ year period with Agriculture, Forestry \& Fishing showing a significant elevation from $0-5$ to $10+$ years and 5-10 to $10+$ years. For ALT Agriculture, Forestry \& Fishing showed a moderately significant elevation from 5-10 to 10+ years. For ALP, Agriculture, Forestry \& Fishing showed a significant increase from $0-5$ to $10+$ years. For GGT there was a moderately significant elevation from $0-5$ to $10+$ years and 5-10 to 10+ years for Agriculture, Forestry \& Fishing. For Mining, there was a moderately significant elevation from $0-5$ to $10+$ years whereas for Construction there was a significantly elevated mean from $0-5$ to $10+$ years. Finally, for total bilirubin there was a significant elevation from $0-5$ to $10+$ years and from $0-5$ to $5-10$ years for Agriculture Forestry \& Fishing. For Construction, there was a moderately significant elevation between $5-10$ years and $10+$ years. This potentially indicates that exposure over a period of time may have varying effects, with some time periods being more detrimental. It must be noted that, with liver injury being multifactorial, lead may not play the largest role and other factors such as alcohol use, drug use, and genetic background, also contribute to liver injury [27].

\subsection{On Lead and Its Role in Altering Hepatobiliary Clinical Makers}

According to Lanphear and co-authors, low-level environmental lead exposure is a factor for diseases such as cardiovascular disease $[28,29]$. It also seems to potentially affect the hepatic and biliary system. In the United States, low-level lead exposure is the norm in many communities because of the legacy of lead exposure, which keeps populations continuously exposed. In addition, the workplace serves as the primary avenue by which adults are exposed.

Results from this study point to the fact that participants at different binary levels of exposure have varied presentations. For example, when lead was divided at $2 \mu \mathrm{g} / \mathrm{dL}$ for less-exposed/exposed, those exposed to lead were more likely to have high ALP (adjusted odds ratio (OR) 1.93; 95\% confidence interval (CI) 1.42, 2.61) and GGT (adjusted OR 1.24; 95\% CI 1.08, 1.42).

At the $5 \mu \mathrm{g} / \mathrm{dL}$ level, those exposed to lead were moderately significantly more likely to have higher ALP (adjusted OR 2.90; 95\% CI 1.18, 7.08), and GGT (adjusted OR 1.48; 95\% CI 1.12, 1.95) levels. At the $10 \mu \mathrm{g} / \mathrm{dL}$ level those exposed to lead were more likely to have higher GGT levels (adjusted OR $2.19 ; 95 \%$ CI 1.34, 3.56). This may indicate lead's effects at different degrees of exposure with damage being manifested differently at various cutoffs.

This study found positive significant associations between BLL and ALP, GGT and total bilirubin. Regarding the exposed and less-exposed, for AST there was a significant elevation from quartile 1 to quartile 2. For ALP there was a significant elevation from quartile 1 to quartile 2 and 3. Finally, for GGT there was a significant elevation from quartile 1 to quartiles 2 and 3. This speaks to increasing 
lead dose producing worse outcomes and that hepatobiliary clinical makers at different degrees of exposure are potential predators of poor health outcomes and all-cause mortality as they reflect liver injury and oxidative stress.

The effects of lead on AST and ALT have been demonstrated in the literature. Onyeneke and co-authors [24] in their study of 86 adult Nigerians who were occupationally exposed to lead and 30 control subjects who were not exposed to lead (while looking to understand occupational exposure to lead in Nigeria and its relation with impairment of liver function), found that the activity of ALT and AST were significantly elevated in occupationally exposed workers as compared to controls, whereas there was no statistically significant changes in serum total bilirubin.

Regarding GGT and its association with BLL in adults, Lee and co-authors in an analysis of NHANES III found associations between blood lead and GGT in adults [30]. Al-Neamy and co-authors [18] in their study found mean AST levels to be elevated in the exposed (31.8 \pm 12.3$)$ as compared to mean AST levels of the less-exposed (30.7 \pm 13.2$)$, however, the means were not statistically significantly different.

For ALT, they found levels to be slightly elevated in the less-exposed ( $33.5 \pm 24.2)$ as compared to the exposed $(33.2 \pm 27.6)$ but the difference was again not statistically significant. The authors found the mean total bilirubin levels to be more elevated in the exposed $(0.75 \pm 0.3)$ compared to the less-exposed $(0.70 \pm 0.19)$ but these differences were not statistically significant. In addition, they found GGT to be elevated in the exposed $(36.3 \pm 35.2)$ as compared to the less-exposed (31.36 \pm 17.9$)$, but the difference was not statistically significant. Finally, they found ALP to be more elevated in the exposed $(84.3 \pm 24.6)$ as compared to the less-exposed $(76.1 \pm 20.5)$ with the difference proving to be statistically significant. Thus, the results of our study, which demonstrates a statistically significant difference in mean GGT levels between the differentially exposed individuals, contradict those of Al-Neamy and co-authors' study while the positive association found in regression affirms that of Lee and co-authors' study. However, our study finding of a significant difference in the means of ALP between the varying degrees of exposure affirms the results of Al-Neamy and co-authors' study.

An observation of the results of our study and those of the others show the uniqueness of our study, given that it looked at larger sample sizes as compared to the non-NHANES studies. Another difference is the fact that, the studies by Onyeneke [24] and Al-Neamy [18] were in Nigeria and the UAE, whereas our analysis was performed on populations in the United States.

Finally, our results regarding ALP, GGT, and total bilirubin indicate significant lead induced pathology in the extrahepatic biliary system, potentially regarding cholestasis, as compared to the intrahepatic system, as there are significant elevations and associations in the aforementioned enzymes as compared to ALT and AST. It should be noted that even though the results regarding ALP and GGT would strongly suggest lead induced hepatobiliary dysfunction, elevated GGT activity may occur in acute and chronic pancreatitis or even prostatic adenocarcinoma with elevated ALP also occurring in other pathology, indicating that using the absence of skeletal diseases and the absence of placenta induced elevations and pairing it with elevated ALP enzymatic activity should not be considered a clinically specific sign of hepatobiliary dysfunction [31].

\subsection{Limitations of Study and Future Works}

Measurement of BLLs does not indicate longer-term exposure, rather, it is indicative of recent lead exposure as well as lead that has been mobilized from bone or other tissue sources with no ability to distinguish between both. Measuring of bone lead levels, particularly tibia lead level, via K-Shell X-Ray Fluorescence (KSXF) would have provided more information on length of exposure as bone lead levels are indicative of long-term cumulative exposure to lead. Both the BLLs and bone lead levels taken together would have provided the best and most comprehensive view of the participant's exposure [32]. In attempting to overcome the limitation of long-term exposure, length of time at occupation was analyzed and seeing the differences in health outcomes overtime helped to give hints on the manifestations of long term exposure. 
Finally, owing to the inability to adjust for covariates in the occupational analysis, and the inability to perform regression analysis on lead exposed occupations due to inadequate data in all strata for any of exposed occupations, future works should look at larger occupational databases. This will enable the evaluation of the significances found here in adjusted models for lead exposed occupations.

\section{Conclusions}

Lead exposure was significantly associated with adverse hepatobiliary clinical makers, with higher exposure resulting in worse outcomes. Looking at various degrees of exposure, lead increased the odds of elevated hepatobiliary clinical markers. Finally, occupational exposure may play a role in these outcomes. These findings add to the growing body of evidence that lead exposure may be an important risk factor for liver and gallbladder dysfunction in exposed populations. Based on the above discussion, it is suggested that a critical need exists to test novel interventions capable of mitigating and subsequently eliminating the impact of lead on hepatobiliary health. Studies aimed at interventions that mitigate and/or eliminate the harmful effects of lead on the environment and on human health are still required for successful optimal health management. This is key as Gould and co-authors note that every dollar invested in lead paint hazard prevention yielded $\$ 17$ to $\$ 221$ return or a total saving of \$181-\$269 billion per year [33]. Finally, this study's cross-sectional nature means that it portrays a snapshot in time. A longitudinal study may yield more in-depth results as people's unique circumstances (finances, family, etc.) change, which may result in gaining access to necessary knowledge and preventative measures about lead exposure and hence seeking avenues to mitigate the effects of it on their health and environment. Ultimately, this work can be used to improve public health by working to limit exposure to higher doses as this seems to be associated with worse liver/biliary injury but, as injury occurs at even low exposure levels it must be emphasized that no level of exposure is safe. Future works should look at individual, community, state, and regional-level risk factors of lead exposure on hepatobiliary clinical markers to better understand how these factors may differentially alter them and the impact at all four levels.

Acknowledgments: We acknowledge the National Center for Health Statistics of the U.S. Centers for Disease Control and Prevention (CDC) for its invaluable work conducting the National Health and Nutrition Examination Survey, and the researchers at the Division of Laboratory Sciences, National Center for Environmental Health of the CDC.

Author Contributions: Emmanuel Obeng-Gyasi conceived the idea for the paper, wrote the paper, and did the analysis. Rodrigo Armijos edited the paper and contributed to the analysis of the data. Margaret Weigel edited the paper and provided analytical evaluation. Gabriel Filippelli provided analytical guidance for the paper. M. Aaron Sayegh provided analytical guidance for the paper.

Conflicts of Interest: The authors declare no conflict of interest.

\section{References}

1. Centers for Disease Control and Prevention. Very high blood lead levels among adults-United States, $2002-2011$. MMWR. Morb. Mort. Wkly Rep. 2013, 62, 967-971.

2. Jarup, L. Hazards of heavy metal contamination. Br. Med. Bull. 2003, 68, 167-182. [CrossRef] [PubMed]

3. Mielke, H.W.; Laidlaw, M.A.; Gonzales, C. Lead (Pb) legacy from vehicle traffic in eight California urbanized areas: Continuing influence of lead dust on children's health. Sci. Total Environ. 2010, 408, 3965-3975. [CrossRef] [PubMed]

4. Johnson, S.; Saikia, N.; Sahu, M.R. Lead in Paints; Centre for Science and Environment, and Pollution Monitoring Laboratory: New Delhi, India, 2009.

5. Díaz-Barriga, F.; Batres, L.; Calderón, J.; Lugo, A.; Galvao, L.; Lara, I.; Rizo, P.; Arroyave, M.E.; McConnell, R. The El Paso smelter 20 years later: Residual impact on Mexican children. Environ. Res. 1997, 74, 11-16. [CrossRef] [PubMed]

6. Oldereid, N.B.; Thomassen, Y.; Attramadal, A.; Olaisen, B.; Purvis, K. Concentrations of lead, cadmium and zinc in the tissues of reproductive organs of men. J. Reprod. Fertil. 1993, 99, 421-425. [CrossRef] [PubMed] 
7. Navas-Acien, A.; Guallar, E.; Silbergeld, E.K.; Rothenberg, S.J. Lead exposure and cardiovascular disease: A systematic review. Environ. Health Perspect. 2007, 115, 472-482. [CrossRef] [PubMed]

8. Dioka, C.E.; Orisakwe, O.E.; Adeniyi, F.A.A.; Meludu, S.C. Liver and renal function tests in artisans occupationally exposed to lead in mechanic village in Nnewi, Nigeria. Int. J. Environ. Res. Public Health 2004, 1, 21-25. [CrossRef] [PubMed]

9. Bellinger, D.; Leviton, A.; Waternaux, C.; Needleman, H.; Rabinowitz, M. Longitudinal analyses of prenatal and postnatal lead exposure and early cognitive development. N. Engl. J. Med. 1987, 316, 1037-1043. [CrossRef] [PubMed]

10. Darwish, W.S.; Ikenaka, Y. Biological responses of xenobiotic metabolizing enzymes to lead exposure in cultured H4IIE rat cells. Jpn. J. Vet. Res. 2013, 61, S48-S53. [PubMed]

11. Hsu, P.-C.; Guo, Y.L. Antioxidant nutrients and lead toxicity. Toxicology 2002, 180, 33-44. [CrossRef]

12. Kim, Y.J. Interpretation of liver function tests. Korean J. Gastroenterol. Taehan Sohwagi Hakhoe chi 2008, 51, 219-224. [PubMed]

13. Centers for Disease Control and Prevention. Summary Health Statistics Tables for US Adults: National Health Interview Survey 2015, Table A-4b. Available online: https:/ ftp.cdc.gov / pub/Health_Statistics / NCHS/NHIS/SHS/2015_SHS_Table_A-4.pdf (accessed on 1 April 2017).

14. Green, R.M.; Flamm, S. AGA technical review on the evaluation of liver chemistry tests. Gastroenterology 2002, 123, 1367-1384. [CrossRef] [PubMed]

15. Johnston, D.E. Special considerations in interpreting liver function tests. Am. Fam. Physician 1999, 59, 2223-2232. [PubMed]

16. Washington, I.M.; Van Hoosier, G. Clinical biochemistry and hematology. In The Laboratory Rabbit, Guinea Pig, Hamster, and Other Rodents; Elsevier: Amsterdam, The Netherlands, 2012; pp. 57-116.

17. Giannini, E.G.; Testa, R.; Savarino, V. Liver enzyme alteration: A guide for clinicians. Can. Med. Assoc. J. 2005, 172, 367-379. [CrossRef] [PubMed]

18. Al-Neamy, F.R.M.; Almehdi, A.M.; Alwash, R.; Pasha, M.A.H.; Ibrahim, A.; Bener, A. Occupational lead exposure and amino acid profiles and liver function tests in industrial workers. Int. J. Environ. Health Res. 2001, 11, 181-188. [CrossRef] [PubMed]

19. Papanikolaou, N.C.; Hatzidaki, E.G.; Belivanis, S.; Tzanakakis, G.N.; Tsatsakis, A.M. Lead toxicity update. A brief review. Med. Sci. Monit. 2005, 11, RA329-RA336. [PubMed]

20. National Center for Health Statistics (NCHS). National Health and Nutrition Examination Survey Questionnaire (or examination protocol, or laboratory protocol). 2008. Available online: https:/ /wwwn.cdc. gov/nchs/nhanes / ContinuousNhanes/Default.aspx?BeginYear=2007 (accessed on 31 March 2018).

21. National Center for Health Statistics (NCHS). National Health and Nutrition Examination Survey Questionnaire (or examination protocol, or laboratory protocol). 2010. Available online: https:/ /wwwn.cdc. gov/nchs/nhanes / ContinuousNhanes/Default.aspx?BeginYear=2009 (accessed on 31 March 2018).

22. Sakai, T. Biomarkers of lead exposure. Ind. Health 2000, 38, 127-142. [CrossRef] [PubMed]

23. Geraldine, M.; Venkatesh, T. Influence of minerals on lead-induced alterations in liver function in rats exposed to long-term lead exposure. J. Hazard. Mater. 2009, 166, 1410-1414.

24. Onyeneke, E.C.; Omokaro, E.U. Effect of Occupational Exposure to Lead on Liver Function Parameters. Int. J. Pharm. Med. Sci. 2016, 6, 15-19.

25. National Center for Health Statistics. National Health and Nutrition Examination Survey (NHANES) Analytic Guidelines; US Department of Health and Human Services: Washington, DC, USA, 2007.

26. Waller, K.; Osorio, A.M.; Maizlish, N.; Royce, S. Lead exposure in the construction industry: Results from the California Occupational Lead Registry, 1987 through 1989. Am. J. Public Health 1992, 82, 1669-1671. [CrossRef] [PubMed]

27. Van Beek, J.H.; de Moor, M.H.; de Geus, E.J.; Lubke, G.H.; Vink, J.M.; Willemsen, G.; Boomsma, D.I. The genetic architecture of liver enzyme levels: GGT, ALT and AST. Behav. Genet. 2013, 43, 329-339. [CrossRef] [PubMed]

28. Lanphear, B.P.; Hornung, R.; Khoury, J.; Yolton, K.; Baghurst, P.; Bellinger, D.C.; Canfield, R.L.; Dietrich, K.N.; Bornschein, R.; Greene, T.; et al. Low-level environmental lead exposure and children's intellectual function: An international pooled analysis. Environ. Health Perspect. 2005, 113, 894-899. [CrossRef] [PubMed]

29. Lanphear, B.P.; Rauch, S.; Auinger, P.; Allen, R.W.; Hornung, R.W. Low-level lead exposure and mortality in US adults: A population-based cohort study. Lancet Public Health 2018. [CrossRef] 
30. Lee, D.H.; Lim, J.S.; Song, K.; Boo, Y.; Jacobs, D.R., Jr. Graded associations of blood lead and urinary cadmium concentrations with oxidative-stress-related markers in the US population: Results from the Third National Health and Nutrition Examination Survey. Environ. Health Perspect. 2006, 114, 350-354. [CrossRef] [PubMed]

31. Clark, V.L.; Kruse, J.A. Clinical methods: The history, physical, and laboratory examinations. JAMA 1990, 264, 2808-2809.

32. Landrigan, P.J.; Todd, A.C. Direct measurement of lead in bone a promising biomarker. JAMA 1994, 271, 239-240. [CrossRef] [PubMed]

33. Gould, E. Childhood lead poisoning: Conservative estimates of the social and economic benefits of lead hazard control. Environ. Health Perspect. 2009, 117, 1162-1167. [CrossRef] [PubMed]

2018 by the authors. Licensee MDPI, Basel, Switzerland. This article is an open access article distributed under the terms and conditions of the Creative Commons Attribution (CC BY) license (http://creativecommons.org/licenses/by/4.0/). 